\title{
Ação inseticida do extrato de Derris amazonica Killip para Cerotoma arcuatus Olivier (Coleoptera: Chrysomelidae)
}

\author{
Márcio Rodrigo ALECIO ${ }^{1}$, Murilo FAZOLIN², Rosalee Albuquerque COELHO NETTO³, Valdomiro \\ CATANI $^{4}$, Joelma Lima Vidal ESTRELA ${ }^{5}$, Suziane Barros ALVES ${ }^{6}$, Raquel da Silva CORREA7, Romeu de \\ Carvalho ANDRADE NETO ${ }^{8}$, Adriana Dantas GONZAGA?
}

\section{RESUMO}

A abundância e o potencial inseticida de Derris amazonica e a necessidade de controle de Cerotoma arcuatus Olivier (Coleoptera: Chrysomelidae) na cultura do feijāo-caupi (Vigna unguiculata L. Walp) estimularam a realização desta pesquisa, que objetivou avaliar a açáo inseticida do extrato de $D$. amazonica a adultos de $C$. arcuatus em condiçóes de laboratório. Os bioensaios testaram as vias de intoxicação por ingestão de folhas contaminadas, contato com superfície contaminada e aplicaçáo tópica, com delineamento experimental inteiramente casualizado, com quatro repetiçóes. Os valores de mortalidade e consumo foliar dos insetos foram submetidos à análise de regressão, sendo utilizada a análise de Probit para determinação das $\mathrm{CL}_{50}$, da $\mathrm{DL}_{50} \mathrm{e}$ $\operatorname{dos} \mathrm{TL}_{50^{\circ}}$ O extrato de $D$. amazonica, contendo $3,7 \%$ de rotenona, foi tóxico para adultos de $C$. arcuatus via ingestão de folhas contaminadas $\left(\mathrm{CL}_{50}=15,14 \mu \mathrm{L}\right.$ do extrato. $\mathrm{mL}^{-1}$ de água), superfície contaminada $\left(\mathrm{CL}_{50}=0,45 \mu \mathrm{L}\right.$ do extrato. $\left.\mathrm{cm}^{-2}\right)$ e aplicação tópica $\left(\mathrm{DL}_{50}=1,44 \mu \mathrm{L}\right.$ do extrato. $\mathrm{g}^{-1}$ do inseto). Mortalidades de adultos de $C$. arcuatus superiores a $80 \%$ e os menores tempos letais médios foram obtidos na concentração de $5 \%\left(\mathrm{v} \mathrm{v}^{-1}\right)$ do extrato em todos os bioensaios. O consumo foliar de adultos de C. arcuatus foi inversamente proporcional a concentraçáo do extrato quando expostos por via de ingestão foliar ou aplicação tópica, sendo inclusive observada inibição da alimentação dos indivíduos. O extrato de $D$. amazonica é tóxico para $C$. arcuatus e inibe a alimentação dos insetos a partir da concentração de $1 \%\left(\mathrm{v} \mathrm{v}^{-1}\right)$.

PALAVRAS-CHAVE: rotenona, toxicidade, inseticidas botânicos.

\section{Insecticide action of the extract of Derris amazonica Killip for Cerotoma arcuatus Olivier (Coleoptera: Chrysomelidae)}

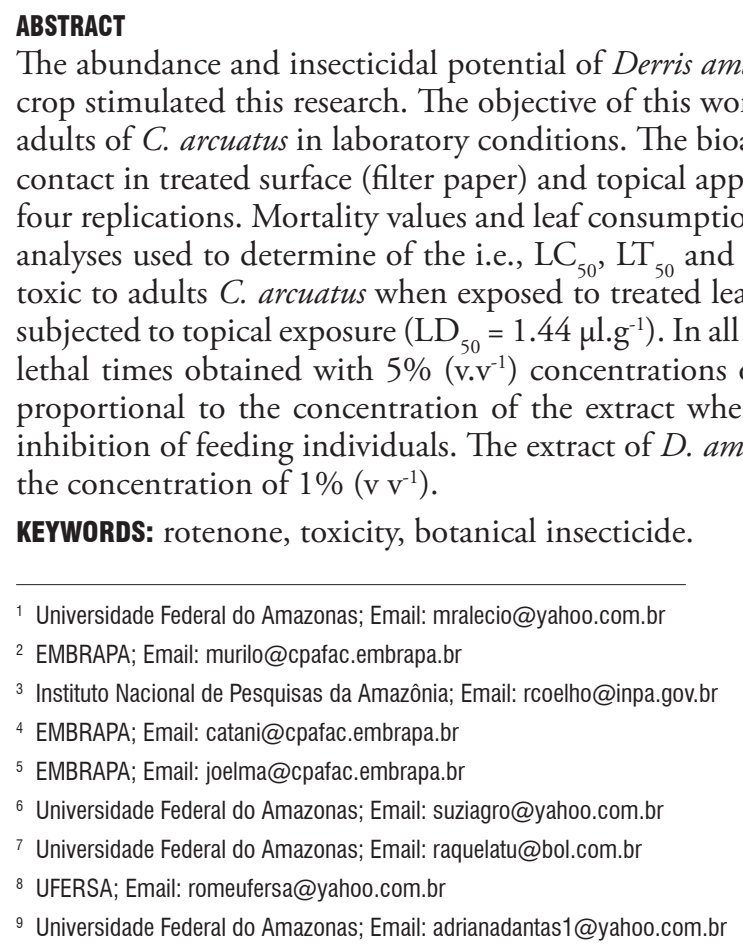
the concentration of $1 \%\left(\mathrm{v} \mathrm{v}^{-1}\right)$.

KEYWORDS: rotenone, toxicity, botanical insecticide.

\footnotetext{
1 Universidade Federal do Amazonas; Email: mralecio@yahoo.com.br

2 EMBRAPA; Email: murilo@cpafac.embrapa.br

3 Instituto Nacional de Pesquisas da Amazônia; Email: rcoelho@inpa.gov.br

${ }^{4}$ EMBRAPA; Email: catani@cpafac.embrapa.br

${ }^{5}$ EMBRAPA; Email: joelma@cpafac.embrapa.br

${ }^{6}$ Universidade Federal do Amazonas; Email: suziagro@yahoo.com.br

7 Universidade Federal do Amazonas; Email: raquelatu@bol.com.br

${ }^{8}$ UFERSA; Email: romeufersa@yahoo.com.br

${ }^{9}$ Universidade Federal do Amazonas; Email: adrianadantas1@yahoo.com.br
}

The abundance and insecticidal potential of Derris amazonica in addition to need of controlling Cerotoma arcuatus for bean crop stimulated this research. The objective of this work was to evaluate insecticide action of the extract of $D$. amazonica to adults of $C$. arcuatus in laboratory conditions. The bioassays were carried out using three distend methodologies: leaf intake, contact in treated surface (filter paper) and topical application. A completed randomized experimental design was used with four replications. Mortality values and leaf consumption of the insects were subjected to regression analyses, being the Probit analyses used to determine of the i.e., $\mathrm{LC}_{50}, \mathrm{LT}_{50}$ and $\mathrm{LD}_{50}$. The extract of $D$. amazonica containing 3.7\% of rotenone was toxic to adults $C$. arcuatus when exposed to treated leaves $\left(\mathrm{LC}_{50}=15.14 \mu \mathrm{l} . \mathrm{mL}^{-1}\right)$, treated surface $\left(\mathrm{LC}_{50}=0.45 \mu \mathrm{l} . \mathrm{cm}^{-2}\right)$ and subjected to topical exposure $\left(\mathrm{LD}_{50}=1.44 \mu \mathrm{l} \cdot \mathrm{g}^{-1}\right)$. In all bioassays the adults mortality was higher than $80 \%$ with lower median lethal times obtained with $5 \%\left(\mathrm{v}^{-1} \mathrm{v}^{-1}\right)$ concentrations of the extract. Leaf consumption by adults $C$. arcuatus was inversely proportional to the concentration of the extract when exposed by leaf intake or topical application, also being observed inhibition of feeding individuals. The extract of $D$. amazonica is toxic to $C$. arcuatus and inhibits the feeding of insects from 


\section{INTRODUÇÃO}

Inseticidas naturais foram largamente utilizados para o controle de pragas até a década de 40, quando, devido ao incremento das pesquisas com agrotóxicos, os produtos sintéticos passaram a ganhar espaço (Pires, 1978).

Entretanto, a contínua utilização do controle químico, com produtos não seletivos e sem rotação, passou a provocar desequilíbrios populacionais de espécies de insetos-praga, mediante a eliminação de insetos benéficos, explosôes populacionais de pragas e, principalmente, perda de eficácia de inseticidas com a seleção de populaçóes resistentes (Kay \& Collins, 1987; Campanhola, 1990; Janprasert et al., 1993; Guedes, 1999; Guedes \& Fragoso, 1999). Adicionalmente, os inseticidas sintéticos passaram a contaminar o ambiente por persistirem na natureza como resíduos tóxicos muito além do tempo desejado (Ndumu, 1999).

Face aos problemas causados pelos inseticidas sintéticos ao ambiente e ao homem surgiu, nas últimas décadas, a percepção e necessidade de se desenvolver tecnologias e produtos para o controle de insetos-praga que sejam eficientes, seguros, seletivos, biodegradáveis, viáveis economicamente e com aplicabilidade em programas de manejo integrado de pragas (Viegas Júnior, 2002).

Compostos oriundos do metabolismo secundário de plantas despontam como fontes potenciais para a produçáo de novos inseticidas (Villalobos, 1996) e que podem atender aos requisitos de eficácia, seletividade e biodegradabilidade (Isman, 2006).

O Brasil é um país mega-biodiverso, possui um grande número de ecossistemas, espécies que interagem dentro destes ecossistemas, e de alelos que diferenciam os indivíduos em cada populaçáa de cada espécie (Clement, 2001). Neste sentido, a biodiversidade da floresta amazônica destaca-se por apresentar um elevado potencial para a produção de compostos secundários de plantas (Pletsch \& Sant'ana, 1995) com ação inseticida.

As espécies de timbó de maior importância pertencem aos gêneros Derris e Lonchocarpus (Costa et al., 1986; Lima, 1987) e, devido a sua toxicidade apresentam comprovada açáo inseticida (Luitgards-Moura et al., 2002; Pereira \& Famadas, 2004; Azevedo et al., 2005) e são utilizadas há várias décadas pelos índios da Amazônia nas pescarias e para o controle de insetos-praga (Hien et al., 2003; Marinos et al., 2004; Kotze et al., 2006). A obtenção, manipulação e aplicação do extrato de timbó é relativamente simples e de baixo custo (Costa $e t$ al., 1999), o que poderá viabilizar o seu uso como inseticida natural.

A toxicidade das espécies Derris e Lonchocarpus é atribuída, principalmente, a rotenona que é um isoflavonóide cristalino, inodoro e insípido, biossintetizado pela via do metabolismo secundário e que aparece sempre acompanhado de outros compostos flavonoídicos rotenóides, tais como, deguelina, tefrosina e toxicarol (Lima, 1987; Lima \& Costa, 1998). A rotenona causa morte dos animais por meio da inibição da cadeia respiratória mitocondrial, bloqueando a fosforilaçáo do ADP a ATP, sendo peixes e insetos altamente sensíveis (Mascaro et al., 1998).

Diversos trabalhos (Costa et al., 1986; Luitgards-Moura et al., 2002; Azevedo et al., 2005) foram realizados visando ao controle de insetos-praga nas últimas décadas, tanto com extratos desse vegetal quanto com a rotenona, seu principal princípio ativo.

Na regiāo Amazônica brasileira, crisomelídeos do gênero Cerotoma têm sido relatados como as principais pragas dos feijoeiros, destacando-se a espécie Cerotoma arcuatus Olivier (Coleoptera: Chrysomelidae) como a principal praga do feijão-caupi (Vigna unguiculata L. Walp), por atacá-lo desde a semeadura até a colheita, reduzindo substancialmente sua produtividade (Fazolin \& Gomes, 1993; Stone \& Sartorato, 1994; Yokoyama, 2006).

Entretanto, não há nenhum produto registrado no Ministério da Agricultura, Pecuária e Abastecimento MAPA para o controle desta praga na cultura do feijáocaupi (MAPA, 2010). Mesmo assim, é comum observar agricultores utilizando inseticidas químicos sintéticos de forma indiscriminada para o controle desses insetos, tais como, beta-ciflutrina (piretróide), imidacloprido (neonicotinóide) e carbosulfano (metilcarbamato de benzofuranila), que são produtos registrados, de acordo com o MAPA (2010), apenas para o controle de $C$. arcuatus na cultura do feijão comum (Phaseolus vulgaris L.).

Desse modo, a abundância de $D$. amazonica na regiáo Amazônica, aliada à importância econômica das vaquinhas-dofeijoeiro e a necessidade de se desenvolver métodos alternativos para o controle de pragas estimularam a realização deste estudo, que teve como objetivo avaliar a ação inseticida do extrato de $D$. amazonica à adultos de $C$. arcuatus.

\section{MATERIAL E MÉTODOS}

Os experimentos foram conduzidos no laboratório de entomologia agrícola do Instituto Nacional de Pesquisas da Amazônia - INPA, em Manaus, AM. As raízes das plantas de $D$. amazonica foram coletadas no município de Presidente Figueiredo-AM e os adultos de $C$. arcuatus foram capturados, com rede entomológica, em áreas cultivadas com feijão-caupi.

\section{Processamento e obtenção do extrato de raízes de D. amazonica}

Raízes de D. amazônica foram coletadas em campo, acondicionadas em sacos plásticos escuros e transportadas ao laboratório para processamento. O material vegetal foi lavado, 
reduzido a pedaços menores e seco em estufa com circulação de ar forçado, a temperatura de $42{ }^{\circ} \mathrm{C}$ por sete dias, até atingir peso constante. Após a secagem foi triturado em moinho tipo faca até ser reduzido a pó.

Para obtenção do extrato, $40 \mathrm{~g}$ de pó de raízes de timbó foram colocados em filtro de papel para café e, juntamente com $200 \mathrm{~mL}$ de álcool etílico 95\% p.a., foram levados ao sistema extrator Soxhlet. Este sistema foi aquecido à temperatura de refluxo do solvente $\left(72 \pm 2^{\circ} \mathrm{C}\right)$ por um período de cinco horas consecutivas. Em seguida, a solução etanólica foi retirada do sistema Soxhlet e levada ao evaporador-rotativo, durante quarenta minutos, para eliminaçáo do solvente. Posteriormente, o extrato foi acondicionado em recipiente de vidro âmbar fechado e protegido com uma embalagem plástica escura, contra a incidência direta de luminosidade. $\mathrm{O}$ extrato foi mantido em geladeira a uma temperatura média de $5^{\circ} \mathrm{C}$, até a sua utilização nos bioensaios. $\mathrm{O}$ rendimento do extrato em relação à massa vegetal seca das raízes de timbó foi de 3,86 \%.

$\mathrm{O}$ teor de rotenona do extrato foi quantificado por meio de análise cromatográfica, utilizando-se cromatógrafo liquido de alta eficiência (CLAE) Shimadzu', equipado com bomba de alta pressão LC-10ATVP, válvula de gradiente quaternário, degaseificador de membrana a vácuo, câmara de mistura para alta e baixa pressão, amostrador automático, detector de ultravioleta com fonte de deutério e comprimento de onda fixado a 295 nm, máximo de absorção da rotenona.

A coluna de análise foi a de fase reversa C-18 modelo Shimpack VP-ODS com 4,6 $\mathrm{mm}$ x $25 \mathrm{~cm}$ e diâmetro da partícula de $5 \mu \mathrm{c}$, mantida a temperatura constante de $25^{\circ} \mathrm{C}$ durante toda a análise, em forno de colunas. Foi utilizada como fase móvel uma mistura de acetonitrila (Merck-LiChrosolv") e água ultrapura obtida em aparelho Millipore a um fluxo constante de $1 \mathrm{~mL}$ por minuto com o seguinte gradiente: início com acetonitrila e água na proporção de $25 / 75\left(\mathrm{v} \mathrm{v}^{-1}\right)$ chegando em 15 minutos a proporçấo de 80/20 $\left(\mathrm{v} \mathrm{v}^{-1}\right)$, sendo mantida constante por 10 minutos. Antes de cada corrida o sistema foi estabilizado por 10 minutos com acetonitrila/água na proporçấo de $25 / 75\left(\mathrm{v} \mathrm{v}^{-1}\right)$.

Os padrôes com $0,1 \mathrm{a} 0,5 \mathrm{mg} \cdot \mathrm{L}^{-1}$ foram preparados por diluição de uma solução estoque de rotenona $\left(1 \mathrm{mg} \cdot \mathrm{L}^{-1} \mathrm{em}\right.$ acetonitrila) em fase móvel (acetonitrila/água 1:1 $-\mathrm{v} \mathrm{v}^{-1}$ ). As amostras para análise foram preparadas por agitaçáo (10 minutos) de $3 \mathrm{~mL}$ de uma solução de $1 \mathrm{mg} \cdot \mathrm{mL}^{-1}$ do extrato bruto com $200 \mathrm{mg}$ de poliamida-6 (Fluka, 0,25 g. $\mathrm{mL}^{-1}$, tamanho de partícula de 50-160 $\mu \mathrm{m}$ ) seguida de filtraçáo em filtro de amostra de 2,5 $\mu \mathrm{m}$. Todas as amostras foram preparadas protegidas de luminosidade e teor de rotenona presente no extrato de D. amazonica foi de $3,71 \%$.

\section{Montagem dos bioensaios}

Para todos os procedimentos experimentais foram utilizados insetos padronizados de acordo com a idade e o tamanho $(5 \pm 1 \mathrm{~mm})$, os quais foram distribuídos em placas de Petri de $7 \mathrm{~cm}$ de diâmetro mantidas em câmaras climatizadas, tipo B.O.D., a temperatura de $27 \pm 5^{\circ} \mathrm{C}$, fotofase de 12 horas e umidade relativa de $65 \pm 10 \%$.

Foram utilizados cinco insetos por placa de Petri para todos os bioensaios; tempo de exposiçáo ao produto de 72, 48 e 72 horas, respectivamente para as vias de intoxicação por ingestão de folhas contaminadas, superfície contaminada (papel-filtro) e aplicação tópica; volume de $2 \mathrm{~mL}$ para pulverização (ingestão de folhas contaminadas), $0,7 \mathrm{~mL}$ para cada papel-filtro de $7 \mathrm{~cm}$ de diâmetro (superfície contaminada) e $1 \mu \mathrm{L}$ aplicado com auxílio de uma micro-seringa graduada na face ventral do pronoto de cada indivíduo e tempo de três minutos para paralisação dos movimentos dos insetos por congelamento (aplicação tópica). Tais determinaçóes foram obtidas a partir de observaçóes preliminares.

As concentrações do extrato de $D$. amazonica utilizadas nos experimentos foram obtidas por meio de bioensaios para obtenção de uma ampla faixa resposta de mortalidade, ou seja, foram determinadas quais as concentraçóes do extrato que provocaram baixa (próximo a $0 \%$ ) e alta (próximo a $100 \%$ ) mortalidade dos indivíduos, segundo as três formas de exposição dos insetos ao produto.

Para isso, $10 \mathrm{~mL}$ da soluçáo-estoque foram diluídos em $40 \mathrm{~mL}$ de álcool etílico p.a.. Em seguida, essa solução foi diluída em água destilada para os bioensaios de ingestâo de folhas contaminadas e superfície contaminada e em acetona p.a. para o bioensaio de aplicação tópica. As proporçôes utilizadas em todos os casos foram de 0:80, 1:79, 1:39, 1:19, 1:9, 1:5, 1:3 e 1:1 que correspondem, aproximadamente, às concentraçôes de $0 \%$ (controle), $0,25 \%$, $0,5 \%, 1 \%, 2 \%, 3,3 \%, 5 \%$ e $10 \%\left(\mathrm{v} \mathrm{v}^{-1}\right)$, respectivamente. Dentro desta faixa de concentraçóes testadas foram selecionadas seis concentraçôes do extrato de $D$. amazônica: $0,5 \%, 1 \%, 2 \%$, $3 \%, 4 \%$ e $5 \%\left(\mathrm{v} \mathrm{v}^{-1}\right)$ que, juntamente com uma testemunha (solvente), formaram os sete tratamentos utilizados nesta pesquisa.

\section{Superfície contaminada (papel-filtro)}

Discos de papel-filtro, com $7 \mathrm{~cm}$ de diâmetro, foram impregnados com $0,7 \mathrm{~mL}$ das diferentes concentraçôes do extrato de $D$. amazônica e secos ao ar por cerca de 2 minutos. Posteriormente foram colocados em placas de Petri e em seguida infestados com cinco adultos de $C$. arcuatus, que permaneceram sem alimentação, constituindo uma parcela experimental. O delineamento foi inteiramente casualizado com quatro repetiçóes, sendo avaliada a mortalidade dos indivíduos a cada oito horas, durante 48 horas. 
Cada bioensaio foi repetido três vezes e os valores de mortalidade dos insetos foram corrigidos pela mortalidade da testemunha, utilizando-se a fórmula de Abbott (1925). Esses valores foram submetidos à análise de Probit, utilizando-se o programa de análises estatísticas SAS (SAS Institute, 1989).

Foram determinadas as curvas de concentraçáo-mortalidade com probabilidade de causar $50 \%$ de mortalidade $\left(\mathrm{CL}_{50}\right)$, de acordo com a metodologia descrita por Finney (1971).

\section{Aplicação tópica}

Adultos de C. arcuatus foram imobilizados por congelamento em freezer $\left(-16^{\circ} \mathrm{C}\right)$ por três minutos e receberam, por meio de uma micro-seringa graduada, $1 \mu \mathrm{l}$ das diferentes concentraçóes do extrato de $D$. amazônica na face ventral do pronoto. No tratamento controle foi aplicado em cada inseto apenas o solvente (acetona). Em cada placa de Petri foram mantidos cinco insetos adultos, que receberam como alimento, diariamente, uma folha de feijão-caupi livre de quaisquer contaminantes químicos, previamente mergulhadas por dois segundos em hipoclorito de sódio a 1\%, com a finalidade de eliminar além da poeira, microorganismos indesejáveis.

O procedimento experimental foi o mesmo adotado para avaliaçáo do efeito por contato em superfície contaminada, sendo avaliada a mortalidade dos insetos a cada 12 horas, durante 72 horas de duração dos experimentos. De posse dos dados diários de mortalidade foram determinadas a dose letal média $\left(\mathrm{DL}_{50}\right)$ e o tempo letal médio $\left(\mathrm{TL}_{50}\right)$ das diferentes concentraçôes do extrato de $D$. amazonica. Além disso, foi avaliado o consumo médio diário dos insetos sobreviventes em folhas de feijão-caupi, por meio da medição da área foliar remanescente, utilizando-se o integrador de área foliar ADC AM-200 (ADC BioScientific Ltd.).

As parcelas experimentais, número de repetiçóes, delineamento estatístico e procedimentos para análise visando o cálculo da $\mathrm{DL}_{50} \mathrm{eTL}_{50}$ seguiram a metodologia descrita para avaliação do efeito por contato com superfície contaminada.

\subsubsection{Ingestâo de folhas contaminadas}

Folhas de feijão-caupi, livres de contaminantes químicos, foram padronizadas de acordo com o tamanho $(7,0 \pm 1,0 \mathrm{~cm}$ de comprimento) e coloração (verde claro). Em seguida, foram mergulhadas em hipoclorito de sódio a $1 \%$ e em água destilada por dois segundos, com a finalidade de eliminar poeira e microrganismos indesejáveis, sendo depositadas sobre papel toalha para eliminação do excesso da solução desinfetante.

Posteriormente, utilizando-se pulverizadores manuais com capacidade para $500 \mathrm{~mL}$, ajustados para liberaçáo uniforme do produto, quatro folhas de feijấo-caupi foram pulverizadas a uma distância média de $35 \mathrm{~cm}$, com $2 \mathrm{~mL}$ das diferentes concentrações do extrato de $D$. amazonica, distribuídos nas duas faces das folhas. No tratamento controle, as folhas foram pulverizadas apenas com água destilada. Cada folha teve seu pecíolo envolvido por um chumaço de algodáo umedecido em água destilada, visando evitar o ressecamento, sendo transferida para uma placa de Petri e infestada com cinco adultos de $C$. arcuatus, constituindo uma parcela experimental.

Foi adotado o mesmo procedimento experimental utilizado para avaliaçáo do efeito por contato com superfície contaminada, sendo avaliada a mortalidade dos insetos a cada 12 horas, durante 72 horas de duração dos bioensaios. Com os dados diários de mortalidade dos indivíduos foram determinadas a concentração letal média $\left(\mathrm{CL}_{50}\right)$ e o tempo letal médio $\left(\mathrm{TL}_{50}\right)$ das diferentes concentraçóes do extrato de $D$. amazonica. O consumo foliar médio diário dos insetos sobreviventes foi determinado seguindo-se a mesma metodologia utilizada para avaliação do efeito com aplicação tópica.

O delineamento estatístico, as parcelas experimentais, número de repetiçóes e procedimentos para análise visando o cálculo da $\mathrm{CL}_{50}$ e $\mathrm{TL}_{50}$ seguiram a metodologia descrita para avaliação do efeito por contato em superfície contaminada.

\section{RESULTADOS E DISCUSSÃO}

A mortalidade de adultos de $C$. arcuatus por aplicação tópica do extrato de D. amazônica foi maior (85\%) na concentração de $5 \%\left(\mathrm{v} \mathrm{v}^{-1}\right)$ e inferior a $11 \%$ nas concentraçóes de $0,5 \%$ e $1 \%\left(\mathrm{v} \mathrm{v}^{-1}\right)$. As concentraçóes de 3\% e 4\% $\left(\mathrm{v} \mathrm{v}^{-1}\right)$ apresentaram valores de mortalidades intermediárias de 50\% e $65 \%$, respectivamente (Figura 1).

Quando expostos ao extrato de D. amazonica por contato em superfície contaminada, nas concentraçóes de 0,5\%, $1 \%$ e $2 \%\left(\mathrm{v} \mathrm{v}^{-1}\right)$, os valores de mortalidade de $C$. arcuatus permaneceram abaixo de 41\%. Nas maiores concentraçóes $4 \%$ e $5 \%\left(\mathrm{v} \mathrm{v}^{-1}\right)$ os valores de mortalidade observados foram de $70 \%$ e $90 \%$, respectivamente (Figura 1).

No bioensaio de intoxicação por ingestão de folhas contaminadas observaram-se valores médios de mortalidade de $50 \%$ dos insetos na concentraçáo de $2 \%\left(\mathrm{v} \mathrm{v}^{-1}\right)$. Mortalidades superiores a $84 \%$ foram verificadas a partir da concentraçáo de 3\% $\left(\mathrm{vv}^{-1}\right)$ do extrato, com destaque para a concentração de $5 \%\left(\mathrm{vv}^{-1}\right)$ que provocou 100\% de mortalidade dos indivíduos (Figura 1).

Adultos de $C$. arcuatus são mais sensíveis ao extrato de D. amazonica quando ingerem folhas contaminadas, seguido pelas vias de superfície contaminada e aplicação tópica. Essas tendências foram mantidas na determinação toxicológica (Tabela 1), onde foram observados valores das concentraçôes letais calculadas pelas vias de intoxicação por ingestão de folhas contaminadas $\left(\mathrm{CL}_{50}=15,14 \mu \mathrm{L}\right.$ do extrato. $\mathrm{mL}^{-1}$ de água) e superfície contaminada $\left(\mathrm{CL}_{50}=0,45 \mu \mathrm{L}\right.$ do extrato. $\mathrm{cm}^{-2}$ ) e valor da dose letal calculada por aplicação tópica $\left(\mathrm{DL}_{50}\right.$ $=1,44 \mu \mathrm{L}$ do extrato. $\mathrm{g}^{-1}$ do inseto), indicando que o extrato de $D$. amazônica foi tóxico para adultos de C. arcuatus. As concentrações equivalentes às $\mathrm{CL}_{50}$ foram de $1,5 \%\left(\mathrm{v} \mathrm{v}^{-1}\right)$ 


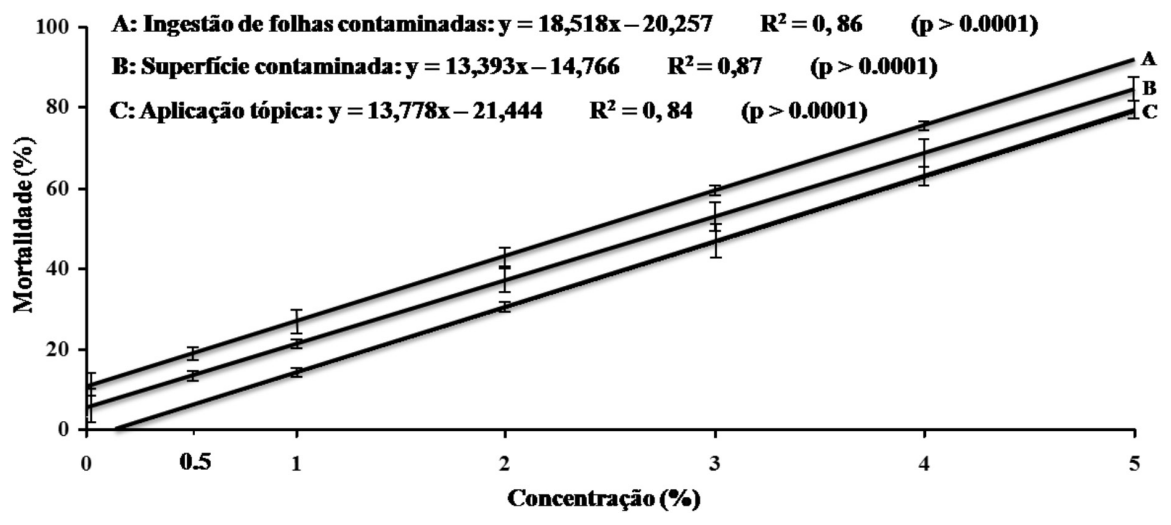

Figura 1 - Ação inseticida do extrato de $D$. amazonica para adultos de $C$. arcuatus por ingestão de folhas contaminadas, superfície contaminada e aplicação tópica.

Tabela 1 - Ação inseticida do extrato de D. amazonica para adultos de C. arcuatus por ingestão de folhas contaminadas, superfície contaminada e aplicação tópica.

\begin{tabular}{|c|c|c|c|c|c|c|c|}
\hline Intoxicação & GL & $n$ & Inclinação \pm EP & $\mathrm{CL}_{50}(\mathrm{IC} 95 \%)$ & $\mathrm{DL}_{50}(\mathrm{IC} 95 \%)$ & $\chi^{2}$ & Prob. \\
\hline $\begin{array}{l}\text { Ingestão de folhas } \\
\text { contaminadas }\end{array}$ & 6 & 420 & $0,95 \pm 0,04$ & $15,14^{*}(11,46-19,03)$ & - & 7,79 & 0,10 \\
\hline Superfície contaminada & 6 & 420 & $0,72 \pm 0,05$ & $0,45^{\star *}(0,39-0,53)$ & - & 6,11 & 0,19 \\
\hline Aplicação tópica & 6 & 420 & $1,03 \pm 0,06$ & - & $1,44^{\star * *} \quad(1,28-1,62)$ & 2,89 & 0,41 \\
\hline
\end{tabular}

GL: graus de liberdade; $n$ : número de insetos usados no bioensaio; $\mathrm{EP}$ : erro-padrão da média; $\mathrm{CL}_{50}$ e $\mathrm{DL}_{50}$ : concentração e dose letal, respectivamente; IC $95 \%$ : intervalo de confiança a $95 \%$ de probabilidade; $\mathrm{X}^{2}$ : qui-quadrado; Prob.: probabilidade; ${ }^{*} \mathrm{CL}_{50}: \mu \mathrm{L}$ do extrato.m- $\mathrm{m}^{-1}$ de agua; ${ }^{\star \star} \mathrm{CL}_{50} ; \mu \mathrm{L}$ do extrato.cm ${ }^{-2} ; * \star \star \mathrm{DL}_{50}: \mu \mathrm{L}$ do extrato. $\mathrm{g}^{-1}$ do inseto.

para ingestão de folhas contaminadas e 2,5\% ( $\left.\mathrm{v} \mathrm{v}^{-1}\right)$ para superfície contaminada e para $\mathrm{DL}_{50}$ foi de $2,9 \%\left(\mathrm{v} \mathrm{v}^{-1}\right)$ por aplicação tópica.

Os valores das inclinaçóes das curvas de concentraçãomortalidade (ingestão de folhas contaminadas e superfície contaminada) e dose-mortalidade (aplicação tópica) (Tabela 1) evidenciaram que adultos de $C$. arcuatus respondem ao extrato de $D$. amazonica pelas três vias de intoxicaçáo. Altos valores de inclinação da curva indicam que pequenas variaçóes na dose do extrato promovem grandes variaçóes na mortalidade dos insetos, resultando em resposta homogênea da população (Atkins et al., 1973).

Resultados semelhantes foram obtidos por Alecio et al. (2005), que obtiveram $\mathrm{CL}_{50}$ de $1,8 \%\left(\mathrm{v} \mathrm{v}^{-1}\right)$ quando avaliaram o potencial inseticida do extrato de Derris rariflora Macbride sobre Sitophilus zeamais Mots (Coleoptera: Curculionidae) pela via de intoxicação de superfície contaminada (papel-filtro). Correa (2006) determinou a toxicidade do extrato de Lonchocarpus floribundus Benth em Toxoptera citricidus Kirkaldy (Sternorrhyncha: Aphididae) por meio de pulverização, sendo a $\mathrm{CL}_{50}$ de $1,8 \%\left(\mathrm{v} \mathrm{v}^{-1}\right)$.
O extrato de $D$. amazonica apresentou elevada toxicidade a adultos de $C$. arcuatus em todas as vias de intoxicação avaliadas, confirmando o potencial inseticida dos timbós (Derris) relatado por diversos pesquisadores nas últimas décadas (Lima \& Costa, 1998; Costa et al., 1999; Luitgards-Moura et al., 2002; Hien et al., 2003; Pereira \& Famadas, 2004; Azevedo et al., 2005; Correa, 2006; Kotze et al., 2006).

O efeito tóxico dos timbós está relacionado principalmente ao teor de rotenona (Crombie \& Whiting, 1998; Fazolin et al., 2002; Marinos et al., 2004; Kotze et al., 2006), cujo valor determinado pela análise cromatográfica foi de 3,7\% no extrato utilizado no presente estudo.

Insetos intoxicados por rotenona apresentam diminuição do consumo de oxigênio, reduçáo da taxa de respiração e ataques que provocam convulsóes e conduzem, finalmente, a paralisias e morte por parada respiratória (Goodman \& Gilman, 1985 e Silva, 2002) Sintomas semelhantes a estes foram obtidos nos resultados deste trabalho, principalmente quando utilizadas maiores concentraçóes do produto $\left(3 \%, 4 \%\right.$ e $\left.5 \% \mathrm{v} \mathrm{v}^{-1}\right)$, sugerindo ser este princípio ativo o principal constituinte tóxico do extrato de $D$. amazonica para $C$. arcuatus. 
Os resultados apontaram a intoxicaçáo por ingestáo de folhas contaminadas como a via causadora da maior toxicidade para adultos de $C$. arcuatus, em todas as concentraçôes do extrato de $D$. amazonica, quando comparadas às mesmas concentraçóes utilizadas no bioensaio de intoxicação por aplicaçáo tópica (Figura 1). Isso pode ter ocorrido pelo fato de, no primeiro caso, os insetos ficarem expostos simultaneamente ao contato tarsal e ingestão do produto tóxico. Segundo Gosselin (1984) estes dois efeitos, progressivamente, são os principais modos de açáo pelo qual a rotenona causa toxidez para insetos.

Silva (2002) relatou que a rotenona é tóxica para insetos por ingestáo e contato, reunindo, de acordo com Cravero et al. (1976), duas formas de intoxicação usadas para controlar insetos-praga (contato e ingestáo). Desse modo, a presença desta substância no extrato de $D$. amazonica poderia justificar a maior toxicidade observada nos bioensaios de ingestáo de folhas contaminadas.

O consumo foliar de adultos de $C$. arcuatus quando alimentados com folhas contaminadas foi menor que aquele obtido nas mesmas concentraçóes do extrato de $D$. amazonica por aplicação tópica (Figura 2). Entretanto, nestas duas condiçôes, as maiores concentraçóes do extrato provocaram os menores valores de consumo foliar dos insetos. Estes valores foram de 2,0 $\mathrm{cm}^{2}$ e $1,8 \mathrm{~cm}^{2}$ por aplicação tópica e de $0,5 \mathrm{~cm}^{2} \mathrm{e}$ $0,4 \mathrm{~cm}^{2}$ por ingestáo de folhas contaminadas, respectivamente para as concentraçóes de $4 \%$ e $5 \%\left(\mathrm{v} \mathrm{v}^{-1}\right)$ do produto.
Adultos de $C$. arcuatus consumiram uma maior área foliar nas primeiras 24 horas dos experimentos independente da forma de exposição ao inseticida botânico, sendo que os indivíduos sobreviventes reduziram, gradativamente, o consumo alimentar com o tempo. Reduçóes bruscas no consumo foliar de adultos de C. arcutus foram observadas quando os mesmos ingeriram folhas contaminadas, principalmente, nas maiores concentraçôes do extrato (3\%, $4 \%$ e $5 \% \mathrm{v} \mathrm{v}^{-1}$ ), ficando os indivíduos sem se alimentarem nas últimas 12 horas de experimentação.

Esses resultados apontaram a ocorrência de inibição de alimentaçâo dos insetos a partir da concentraçấo de $1 \%$ do extrato de $D$. amazonica nas duas vias de intoxicação testadas (aplicaçáo tópica e ingestão de folhas contaminadas), semelhantemente aos efeitos causados pela rotenona extraída dos timbós $D$. elliptica Benth e D. urucu Macbride, relatados, respectivamente nos trabalhos de Nawnot et al. (1987) e Fazolin et al. (2002).

A inibição da alimentação de adultos de $C$. arcuatus observada no presente estudo pode estar relacionada à ocorrência de distúrbios fisiológicos, resultante da toxicidade da rotenona presente no extrato de $D$. amazonica, uma vez que, nas concentraçóes de $2 \%, 3 \%, 4 \%$ e $5 \%$ v v $^{-1}$ (ingestão de folhas contaminadas) e 3\%, 4\% e 5\% v v ${ }^{-1}$ (aplicação tópica) foram obtidos valores diários de consumo menores que $1,4 \mathrm{~cm}^{2}$, abaixo deste determinado por Fazolin \& Estrela (2004) para Cerotoma tingomarianus Bechyné (Coleoptera: Chrysomelidae), em feijoeiro cv. Pérola.

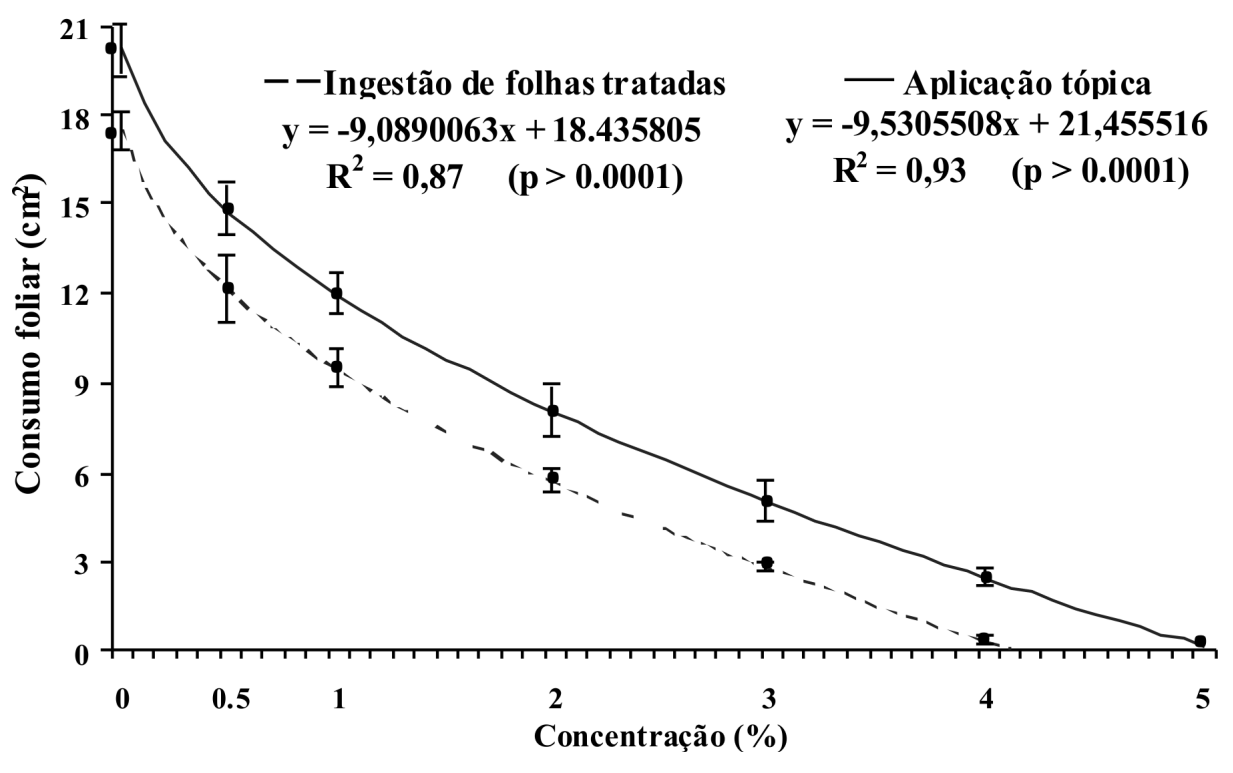

Figura 2 - Consumo foliar de adultos de $C$. arcuatus submetidos ao extrato de $D$. amazonica em diferentes concentrações pelas vias de aplicação tópica e ingestão de folhas contaminadas. 
De acordo com Goodman \& Gilman (1985) a vitamina K3 (menadiona) pode ativar uma rota metabólica alternativa que evita o sítio bloqueado pela açấo da rotenona na respiraçáo, o que permite a retomada do transporte de elétrons e respiração celular dos insetos.

Quando adultos de C. arcuatus foram expostos ao extrato de $D$. amazonica por aplicação tópica, receberam para alimentação folhas de feijāo-caupi, livres de contaminantes, contendo vitamina $\mathrm{k} 3$ conforme Damon et al. (2005), Fugesp (2010) e USDA (2010). Isso pode ter aumentado a capacidade de destoxificação dos indivíduos, principalmente nas menores concentraçôes do extrato $\left(1 \%, 2 \%\right.$ e $\left.3 \% \mathrm{v} \mathrm{v}^{-1}\right)$. Nestas concentraçôes, poucas horas após o início dos experimentos, foram observadas significativas reduçóes na mortalidade dos insetos, o que poderia justificar a hipótese levantada.

Por outro lado, insetos expostos por contato em superfície contaminada sem oferta de alimento podem ter tido uma redução da capacidade de destoxificação, pois mortalidade foi observada durante todo o período de avaliaçáo em todas as concentraçôes do extrato de $D$. amazonica.

Os tempos letais médios $\left(\mathrm{TL}_{50}\right)$ do extrato de $D$. amazonica para adultos de $C$. arcuatus, obtidos pela análise de Probit, apresentam coeficientes angulares positivos, indicando acréscimos nos valores de mortalidade com o tempo de exposiçáo nos três bioensaios (Tabela 2). Entretanto, os menores valores de $\mathrm{TL}_{50}$ foram obtidos no bioensaio de ingestão de folhas contaminadas, seguido pelas vias de superfície contaminada e aplicação tópica. Nestas condiçôes, as maiores concentraçôes do produto apresentaram os menores valores de tempos letais. $\mathrm{Na}$ concentração de $5 \%\left(\mathrm{v} \mathrm{v}^{-1}\right)$ do extrato esses valores variaram de 13,95 a 22,46 horas, em todas as vias de intoxicação.

Resultado semelhante foi obtido por Ribeiro \& Correa (2005), que encontraram o valor de $\mathrm{TL}_{50}$ de 15 horas para concentração tóxica $\left(3,1 \% \mathrm{v} \mathrm{v}^{-1}\right)$ do extrato de $L$. floribundus para Aethalion sp. (Hemiptera: Aethalionidae).

Por ter apresentado os maiores valores de mortalidade e os menores valores de consumo foliar, além dos menores tempos letais médios $\left(\mathrm{TL}_{50}\right)$, pode-se inferir que a intoxicação por ingestão de folhas contaminadas com extrato de $D$. amazonica causou maior toxicidade para $C$. arcuatus.

Contudo, por ter sido tóxico em diferentes níveis, pelas três formas de exposiçấo testadas, o extrato de $D$. amazonica apresenta-se como uma alternativa promissora para ser incluído no manejo integrado de $C$. arcuatus em áreas cultivadas com feijão-caupi na Amazônia brasileira.

Tabela 2 - Tempo letal médio $\left(\mathrm{TL}_{50}\right)$ em horas do extrato de $D$. amazonica a adultos de $C$. arcuatus em diferentes concentrações, expostos pelas vias de superfície contaminada, aplicação tópica e ingestão de folhas contaminadas.

\begin{tabular}{|c|c|c|c|c|c|}
\hline Intoxicação & Concentração & Inclinação \pm EP & $\mathrm{TL}_{50}(\mathrm{IC} 95 \%)$ & $\chi^{2}$ & Prob. \\
\hline \multirow{3}{*}{ Superfície contaminada } & $3 \%$ & $0,48 \pm 0,04$ & $\begin{array}{c}44,07 \\
(34,3-68,6)\end{array}$ & 1,07 & 0,90 \\
\hline & $4 \%$ & $0,64 \pm 0,05$ & $\begin{array}{c}29,32 \\
(24,68-35,86)\end{array}$ & 0,20 & 0,99 \\
\hline & $5 \%$ & $0,65 \pm 0,06$ & $\begin{array}{c}13,95 \\
(10,46-16,96)\end{array}$ & 0,99 & 0,91 \\
\hline \multirow{3}{*}{ Aplicação tópica } & $3 \%$ & $0,61 \pm 0,07$ & $\begin{array}{c}48,91 \\
(38,54-78,26)\end{array}$ & 0,15 & 0,93 \\
\hline & $4 \%$ & $0,62 \pm 0,07$ & $\begin{array}{c}33,63 \\
(26,85-46,60)\end{array}$ & 1,58 & 0,45 \\
\hline & $5 \%$ & $0,70 \pm 0,05$ & $\begin{array}{c}22,46 \\
(17,31-27,09)\end{array}$ & 0,60 & 0,89 \\
\hline \multirow{4}{*}{$\begin{array}{l}\text { Ingestão de folhas } \\
\text { contaminadas }\end{array}$} & $2 \%$ & $0,51 \pm 0,04$ & $\begin{array}{c}68,52 \\
(54,32-102,37)\end{array}$ & 0,22 & 0,99 \\
\hline & $3 \%$ & $0,92 \pm 0,05$ & $\begin{array}{c}31,78 \\
(27,74-35,92)\end{array}$ & 3,95 & 0,41 \\
\hline & $4 \%$ & $0,74 \pm 0,05$ & $\begin{array}{c}20,00 \\
(15,72-23,78)\end{array}$ & 3,80 & 0,43 \\
\hline & $5 \%$ & $0,70 \pm 0,05$ & $\begin{array}{c}15,17 \\
(11,53-18,34)\end{array}$ & 4,24 & 0,37 \\
\hline
\end{tabular}

EP: erro-padrão da média; $\mathrm{TL}_{50}$ : tempo letal; IC 95\%: intervalo de confiança a 95\% de probabilidade; $\mathrm{X}^{2}$ : qui-quadrado; Prob.: probabilidade. 


\section{CONCLUSÃO}

O extrato de $D$. amazonica é tóxico para adultos de $C$. arcuatus, sendo que a via de ingestáo de folhas contaminadas é a mais efetiva, seguida pelo contato com superfície contaminada e aplicação tópica;

Extrato de $D$. amazonica inibe a alimentação de adultos de $C$. arcuatus a partir da concentração de $1 \%$, independente do tipo de exposiçáo do inseto a esse inseticida.

\section{BIBLIOGRAFIA CITADA}

Abbott, W.S. A. 1987. Method of computing the effectiveness of an insecticide. Journal of the American Mosquito Control Association, 3: 302-303.

Alecio, M. R., Alves, S. B., Gonzaga, A. D., Ribeiro, J. D., Correa, R. S. 2005. Evaluation of insecticidal potential in vitro of aqueous extract of the roots of timbo (Derris rariflora) on Sitophilus zeamais Mots In: I Jornada Amazonense de Plantas medicinais. Manaus. Anais da I Jornada Amazonense de Plantas medicinais, p. 42 (in Portuguese).

Atkins, E.L.; Greywood, E.A.; Macdonald, R.L. 1973. Toxicity of pesticides and other agricultural chemicals to honey bees: laboratory studies, 36pp.

Azevedo, F. R.; Guimarães, J. A.; Braga Sobrinho, R.; Lima, M. A. A. 2005. Effciency of natural products to control bemisia tabaci biotype b (hemiptera: aleyrodidae) on melon plant. Revista Arquivos do Instituto Biológico, 72(1): 73-79 (in Portuguese, with abstract in English).

Campanhola, C. 1990. Insect resistance to insecticides: the importance, characteristics and management. Jaguariúna: EMBRAPACNPDA, 45 pp (in Portuguese).

Clement, C.R. 2001. Improvement of native species. In: Nass, L.L.; Valois, A.C.C.; Melo, I.S.; Valadares-Inglis, M.C. (Eds.). Recursos genéticos \& melhoramento de plantas. Fundação de Apoio à Pesquisa Agropecuária de Mato Grosso - Fundação MT, Rondonópolis, MT, p. 423-441 (in Portuguese).

Correa, R. S. 2006. Toxicity of extracts from Lonchocarpus floribundus Benth (timbo) on Toxoptera citricida Kirkald (black citrus aphid) (Sternorrhynda: Aphididae). Dissertação de Mestrado, Instituto Nacional de Pesquisas da Amazônia/Fundaçáo Universidade do Amazonas, Manaus, Amazonas. 71pp (in Portuguese).

Costa, N. A.; Nascimento, C. N. B.; Moura Carvalho, L. O. D.; Pimentel, E. S. 1986. Use of timbo urucu (Derris urucu) in the control of lice (Haemotopinus tuberculatus) in buffalo. Belém: EMBRAPA CPATU, 16 p. (Boletim de Pesquisa e Desenvolvimento, 78) (in Portuguese).

Costa, R.C.L.; Cardoso, B.B.; Silva, J.T.; Gomes Filho, J.G.F.; Silveira, J.A.G. 1996. Water stress strongly decreases the uptake of nitrate and nodulation in bean (Vigna unguiculata (L.) Walp.) In: Reunião Nacional de Pesquisa de Caupi, p. 78-79 (in Portuguese).

Costa, J. P. C.; Alves, S. M.; Belo, M. 1999. Differences among Timbo (Derris spp., Fabaceae) Species from Different Amazonian
Regions in the control of Musca domestica L. Acta Amazônica, 29(4): 573-583 (in Portuguese).

Cravero, E. S.; Guerra. M de S., Silveira, C. P. D. 1976. Manual de inseticidas e acaricidas: aspectos toxicológicos, Pelotas: Aimara, 229 pp (in Portuguese).

Crombie, L.; Whiting, A.D. 1998. Biosynthesis in the rotenoid group of natural products: applications of isotope methodology. Phytochemistry, 49(6): 1479-1507.

Damon, M.; Zhang, N. Z.; Haytowitz, D. B.; Booth, S. L. 2005. Phylloquinone (vitamin $\mathrm{K}_{1}$ ) content of vegetables. Journal of Food Composition and Analysis, 18: 751-758.

Fazolin, M.; Gomes, T.C.A. 1993. Population dynamics of Cerotoma tingomarianus Bechyné in cowpea and kudzu in Rio Branco, Acre. Anais da Sociedade Entomológica do Brasil, 3(22): 491-495 (in Portuguese).

Fazolin, M. 2002. Evaluation of plants with insecticide to control the cow-the-bean. Rio Branco: EMBRAPA-CPAFAC, 42p (Boletim de Pesquisa e Desenvolvimento, 37) (in Portuguese).

Fazolin, M.; Estrela, J. L. V. 2004. Determination of the economic injury level of Cerotoma tingomarianus Bechyné (Coleoptera: Chrysomelidae) on Phaseolus vulgaris L. cultivar Pérola (Coleóptera: Chrysomelidae). Neotropical Entomology, 33: 1-7 (in Portuguese, with abstract in English).

Finney, D. J. 1971. Probit analysis. 3. th. New Delhi: Cambridge University Prees, 333pp.

Fugesp, 2009. Bulário: suplementação vitamínica (www.fugesp.org. br/fugesp_bulario.asp), acesso: 27/05/10.

Goodman, L. S.; Gilman, A. G. 1985. The Pharmacological Basis of Therapeutics. 7th ed. New York, Toronto, London: Macmillan, p. $1839-1856$.

Gosselin, R. E. 1984. Clinical Toxicology of Commercial Products. 5th ed. Baltimore/London: Williams \& Wilkins, p.111-367.

Guedes, R.N.C. 1999. Insect resistance to insecticides . In: Zambolim, L. I Encontro sobre manejo de doenças e pragas. Viçosa: UFV, p. 101-107 (in Portuguese).

Guedes, R. N. C.; Fragoso, D. B. 1999. Insecticide resistance: Bases general, situation and reflections on the phenomenon in insect pest of coffee. In: Encontro sobre produção de café com qualidade. Viçosa, Minas Gerais, p. 99-120 (in Portuguese).

Hien, P.P.; Gortnizka, H.; Kraemer, R. 2003. Rotenone - potential and prospect for sustainable agriculture. Omonrice, 11: 83-92.

Isman, M.B. 2006. Botanical insecticides, deterrents, and repellents in modern agriculture and an increasingly regulated world. Annual Review of Entomology, 51: 45-66.

Janprasert, J.; Satasook, C.; Sukumalanand, P.; Champagne, D. E.; Kay, I. R.; Collins, P. J.; 1993. The Problem of resistance to insecticides in tropical insect pests. Insect Science and its Applications, 8: 715-721.

Kay, I. R.; Collins, P. J. 1987. The Problem of resistance to insecticides in tropical insect pests. Insect Science and its Applications, 8: 715-721.

Kotze, A. C.; Dobson, R.J.; Chandler, D. 2006. Synergism of rotenone by piperonyl butoxide in Haemonchus contortus 
and Trichostrongylus colubriformis in vitro: Potential for drug-synergism through inhibition of nematode oxidative detoxification pathways. Veterinary Parasitology, 136: 275-282.

Lima, R. R. 1987. Information on two species of timbo: Derris urucu (Killip et al Smith) Macbride e Derris nicou (Killip et Smith) Macbride as insecticides plants. Belém: EMBRAPA CPATU, 23 p. (Documentos, 42) (in Portuguese).

Lima, R. R.; Costa, J. P. C. 1998. Collecting plants from pre Columbian culture in the Brazilian Amazon. Belém: EMBRAPA CPATU, 148 p. (Documentos, 99) (in Portuguese).

Luitgards-Moura, J. F.; Bermudez, E. G. C.; Rocha, A. F. I.; Tsouris, P.; Rosa-Freitas, M. G. 2002. Preliminary Assays Indicate that Antonia ovata (Loganiaceae) and Derris amazonica (Papilionaceae), Ichthyotoxic Plants Used for Fishing in Roraima, Brazil, Have an Insecticide Effect on Lutzomyia longipalpis (Diptera: Psychodidae: Phlebotominae). Memórias do Instituto Oswaldo Cruz. Rio de Janeiro, RJ, 97(5): 737-742 (in English).

Marinos, C.; Castro, J.; Nongrados, D. 2004. Biocidal effect del barbasco Lonchocarpus utilis (Smith, 1930) as regulator of mosquitoes larvae. Revista Peruana de Biologia, 11(1): 87-94 (in Spanish, with abstract in English).

MAPA, 2010. Agrofit. (http://agrofit.agricultura.gov.br/agrofitcons/ principalagrofitcons), acesso: 04/04/10.

Mascaro, U. C. P.; Rodrigues, L. A.; Bastos, J. K.; Santos, E.; Costa, J. P. C. 1998. $\mathrm{LD}_{50}$ in fish and rat produced by powdered roots of Derris spp and ecotoxicological implications. Pesquisa Veterinária Brasileira, 18(2): 53-56 (in Portuguese, with abstract in English).

Nawnot, J., Harmatha, J., Bloszy, E. 1987. Secondary plant metabolites with antifeeding activity and their effects on some stored product insects. In: Internacional Working Conference on Stored-Product Protecion. Donahaye, p. 591-597.

Ndumu, P. A. 1999. Agrochemical from Natural Products. Phytoter. Res, New York, p. 532-548.

Pereira, J.R.; Famadas, K.M. 2004. Evaluation "in vitro" of the efficiency of timbo root extract (Dablstedtia pentaphylla) (Leguminosae, Papilionoidae, Millettiedae) on Boophilus microplus (Canestrini, 1887) in the Paraíba Valley region - São Paulo, Brazil. Arquivos do Instituto Biológico. São Paulo, 71(4): 443-450 (in Portuguese, with abstract in English).
Pires, J. M. 1978. Ichthyotoxic plants - botanical aspects. Ciência e Cultura. 32: 37-41 (in Portuguese).

Pletsch, M.; Sant'ana, A. E. G. 1995. Secoundary compound accumulation in plants-The application of plant biotechnology to plant improvement. Chemistry of Amazon, 5: 51-64.

Ribeiro, J. D.; Correa, R. S. 2005. Use of Lonchocarpus floribundus (Timbo) on spittlebugs fruit (Aethalion sp.) in greenhouse. In: XIV Congresso Brasileiro de Toxicologia. Recife-Pe, p. 306 (in Portuguese).

SAS Institute. 1989. SAS User's Guide: Statistics. SAS Institute, 12(8).

Silva, G., A. Lagunes, J. C. Rodríguez y D. Rodríguez. 2002. Vegetable Insecticides: A New-Old Option in Pest Management. Revista Manejo Integrado de Plagas, 2(3): 21-56 (in Spanish, with abstract in English).

Stone, L.F.; Sartorato, A. 1994. The cultivation of beans: technical recommendations. Brasília: EMBRAPA-CNPAF, 83p. (Documentos, 48) (in Portuguese).

Viegas Júnior, C. J. 2003. Terpenes with insecticidal activity: an alternative to chemical control of insects. Quimica Nova, 26(3): 390-400 (in Portuguese, with abstract in English).

USDA, 2010. National Nutrient Database for Standard Reference, Release 20 (http://www.nal.usda.gov/fnic/foodcomp/Data/ SR20/nutrlist/sr20w430.pdf), acesso: 27/05/10.

Villalobos, M. J. P. 1996. Plaguicidas naturales de origen vegetal: Estado actual de la investigacion. Madrid: Ministerio de Agricultura, Pesca Y alimentacion. Monografias INIA, 35 pp.

Yokoyama, M. 2006. Pests. In: Vieira, C.; Paula Junior, T. J.; Borém, A. (Eds). Bean: 2nd edition updated and expanded. Universidade Federal de Viçosa, Viçosa, Minas Gerais, p. $345-$ 346 (in Portuguese).

Recebido em 27/01/2010

Aceito em 03/07/2010 
